\title{
Equilibration of a one-dimensional Wigner crystal
}

\author{
K. A. Matveev, ${ }^{1}$ A. V. Andreev, ${ }^{2}$ and M. Pustilnik ${ }^{3}$ \\ ${ }^{1}$ Materials Science Division, Argonne National Laboratory, Argonne, IL 60439, USA \\ ${ }^{2}$ Department of Physics, University of Washington, Seattle, WA 98195, USA \\ ${ }^{3}$ School of Physics, Georgia Institute of Technology, Atlanta, Georgia 30332, USA
}

(Dated: April 2, 2010)

\begin{abstract}
Equilibration of a one-dimensional system of interacting electrons requires processes that change the numbers of left- and right-moving particles. At low temperatures such processes are strongly suppressed, resulting in slow relaxation towards equilibrium. We study this phenomenon in the case of spinless electrons with strong long-range repulsion, when the electrons form a one-dimensional Wigner crystal. We find the relaxation rate by accounting for the Umklapp scattering of phonons in the crystal. For the integrable model of particles with inverse-square repulsion, the relaxation rate vanishes.

PACS numbers: $71.10 . P m$
\end{abstract}

The low energy properties of systems of interacting fermions in one dimension are commonly described in the framework of the Luttinger liquid theory [1, 2]. This theory successfully predicted a number of interesting properties of one-dimensional electron systems, such the powerlaw renormalization of the tunneling density of states and impurity potential [3, 4]. On the other hand, much recent interest in interacting one-dimensional Fermi systems was focused on the phenomena not captured by the Luttinger liquid theory [5] 8$]$. One example involves equilibration of a moving one-dimensional electron liquid, which was recently shown to affect the conductance of quantum wires [9] and drag between two wires [10].

In the case of weakly interacting one-dimensional electrons the physical mechanism of equilibration was discussed in Ref. [11]. At low temperature $T$ excitations of the system are particle-hole pairs near the two Fermi points, with the typical energy $T \ll E_{F}$ and momentum $T / v_{F} \ll p_{F}$. (Here $E_{F}, v_{F}$, and $p_{F}$ are the Fermi energy, velocity and momentum of the system.) In this regime, backscattering of a right-moving electron near the Fermi level requires transfer of momentum $2 p_{F}$ to a large number of particle-hole pairs. The most efficient such process consists of a sequence of scattering events, in which a hole passes from the left to the right Fermi point through the bottom of the band. Such processes are suppressed as $e^{-E_{F} / T}$ and, consequently, equilibration of the chemical potentials of the right- and left-moving electrons is a very slow process.

Equilibration of one-dimensional fermions beyond the weak interaction regime is a more challenging problem. In this case the description in the language of particles and holes is no longer applicable, and according to the Luttinger liquid theory the elementary excitations of the system are bosons. On the other hand, the Luttinger liquid theory does not adequately describe particles near the bottom of the band, and is therefore incapable of describing the equilibration processes. In this paper we show that this difficulty can be overcome in the limit of strong long-range interactions.

More specifically, we consider a system of identical spinless particles of mass $m$ described by the Hamiltonian of a general form

$$
H=\sum_{l} \frac{p_{l}^{2}}{2 m}+\frac{1}{2} \sum_{l, l^{\prime}} V\left(x_{l}-x_{l^{\prime}}\right) .
$$

Here $x_{l}$ and $p_{l}$ are the coordinate and momentum of the $l$-th particle, and $V(x)$ is the interaction potential. In the limit of very strong repulsion, the particles form a periodic chain with interparticle distance $a=1 / n$ determined by their density $n$. In the case of Coulomb repulsion, $V(x)=e^{2} /|x|$, such an arrangement is commonly referred to as the Wigner crystal.

At strong but finite repulsion, the particles can deviate from their respective lattice sites, $x_{l}=l a+u_{l}$, but the relative change of interparticle distance remains small, $\left|u_{l}-u_{l^{\prime}}\right| \ll\left|l-l^{\prime}\right| a$. To leading order in the deviations $u_{l}$ the Hamiltonian (1) takes the form

$$
H_{0}=\sum_{l} \frac{p_{l}^{2}}{2 m}+\frac{1}{4} \sum_{l, l^{\prime}} V_{l-l^{\prime}}^{(2)}\left(u_{l}-u_{l^{\prime}}\right)^{2}
$$

where we used the following notation for the $r$-th derivative of the interaction potential

$$
V_{l}^{(r)}=\left.\frac{d^{r} V(x)}{d x^{r}}\right|_{x=l a} .
$$

Elementary excitations of the harmonic chain (2) are phonons characterized by quasimomentum $q$ (i.e., $u_{l} \propto$ $\left.e^{i q l}\right)$. Their frequencies are easily found by solving the classical equations of motion,

$$
\omega_{q}^{2}=\frac{2}{m} \sum_{l=1}^{\infty} V_{l}^{(2)}[1-\cos (q l)] .
$$

Provided that the interaction potential $V(x)$ falls off faster than $1 /|x|$ at large distances, the low-energy excitations of the system are bosons with acoustic spectrum 


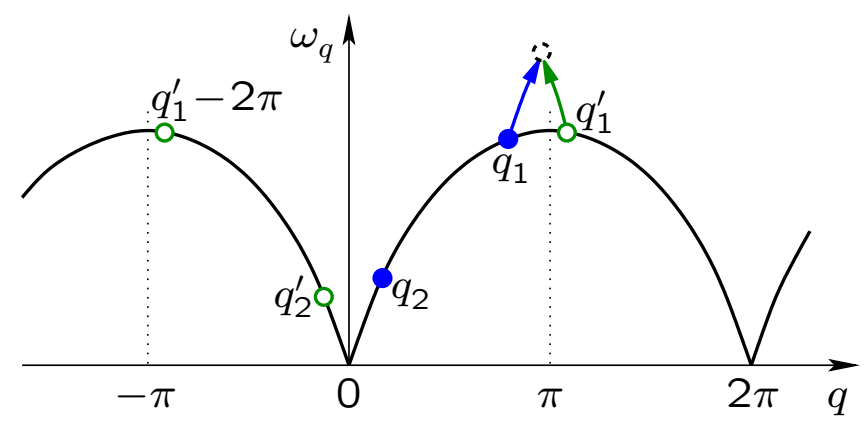

FIG. 1: Umklapp scattering of two right-moving phonons (filled circles) into two left-moving phonons (open circles). The energies of the initial and final states are equal (see the dashed circle). On the other hand, the sum of the quasimomenta defined to range in the first Brillouin zone $-\pi<q<\pi$, decreases by $2 \pi$.

$\omega_{q}=s|q|$, in agreement with the Luttinger liquid theory [12].

Importantly, in the limit of strong interactions the harmonic approximation (2) provides the full spectrum of elementary excitations (4), not limited by the restriction $|q| \ll 1$ imposed in the Luttinger liquid theory. Another advantage of the Wigner crystal model is that the weak interaction of phonons is naturally described the anharmonic terms in the expansion of the Hamiltonian (11) in the powers of the displacements $u_{l}$. Scattering of phonons resulting from these interactions leads to relaxation of their distribution function to equilibrium.

At temperatures $T$ much lower than the Debye energy $\hbar \omega_{\pi}$, the quasimomenta of the thermally excited phonons are small, $q \ll 1$. For such phonons the Umklapp scattering is impossible, and phonon-phonon collisions conserve the total quasimomentum $Q$ of the system. As a result, the equilibrium distribution of the phonons

$$
N_{q}=\frac{1}{e^{\hbar\left(\omega_{q}-u q\right) / T}-1}
$$

is characterized by two parameters: the temperature $T$ and the velocity $u$ of the phonon gas with respect to the lattice (see, e.g., Ref. [13]).

It is important to note that while being a good approximation, conservation of quasimomentum is not exact. Indeed, even at low temperatures there is a finite occupation $N_{\pi} \sim e^{-\hbar \omega_{\pi} / T}$ of phonon states near the edge $q=\pi$ of the Brillouin zone, leading to a small probability of Umklapp processes. As a result, the total quasimomentum of the phonons relaxes as $\dot{Q}=-Q / \tau$ with the time constant $\tau \propto e^{\hbar \omega_{\pi} / T}$. For distribution (5) one has $Q \propto u$, and thus the velocity $u$ acquires a time dependence, $\dot{u}=-u / \tau$. Calculation of the relaxation time $\tau$ is our main goal.

The microscopic mechanism of Umklapp scattering is illustrated in Fig. 1. We assume that the phonon spectrum is concave, which is the case for Coulomb repul- sion. Then the dominant process involves scattering of a rare phonon with quasimomentum $q_{1}$ near the boundary of the Brillouin zone by an acoustic phonon with energy $\hbar \omega_{q_{2}} \sim T$. As a result of such a collision the quasimomentum of the high-energy phonon changes by $\delta q_{1} \sim T / \hbar s$. If the new quasimomentum $q_{1}^{\prime}=q_{1}+\delta q_{1}$ is outside the Brillouin zone $(-\pi, \pi)$, the scattering involves Umklapp, and the total quasimomentum changes by $\pm 2 \pi$.

In the course of such scattering events the number of the rare phonons near the edge of the Brillouin zone is conserved, while their momentum changes by a small amount $\delta q \ll \pi$. Thus these phonons essentially diffuse in the momentum space, and the evolution of their distribution function $P(q, t)$ can be described by the FokkerPlanck equation

$$
\partial_{t} P=-\partial_{q} J, \quad J=A(q) P(q, t)-\frac{1}{2} \partial_{q}[B(q) P(q, t)] .
$$

Here $J$ has the meaning of the probability current in momentum space, and the functions $A(q)$ and $B(q)$ can be expressed in terms of the rate $W_{q, q+\delta q}$ of the phonon transition from state $q$ to $q+\delta q$ as

$$
A(q)=\sum_{\delta q} \delta q W_{q, q+\delta q}, \quad B(q)=\sum_{\delta q}(\delta q)^{2} W_{q, q+\delta q}
$$

Below we will be using the Fokker-Planck equation (6) to determine the behavior of the phonon distribution function $P$ in a small vicinity of $q=\pi$. This enables us to approximate $B(q)$ by $B=B(\pi)$. Furthermore, we will be interested in the case of weak deviation from equilibrium, when the velocity of the phonon system is small, $u \ll s$. Thus we can approximate $A(q)$ by its value at equilibrium, when the Boltzmann distribution $P(q)=e^{-\hbar \omega_{q} / T}$ has to solve the Fokker-Planck equation by nullifying the probability current $J$. Then, from (6) we find $A(q)=-B \hbar \omega_{q}^{\prime} / 2 T$, where $\omega_{q}^{\prime}=d \omega_{q} / d q$.

The phonon distribution function $P(q)$ is $2 \pi$ periodic. In the first Brillouin zone and away from its edges $q= \pm \pi$ it is given by Eq. (5). A periodic continuation of (5) results in a formal discontinuity at $q=\pi$. Specifically, the phonon distribution function away from $q=\pi$ takes the form

$$
P(q)=e^{-\hbar \omega_{q} / T} e^{ \pm \pi \hbar u / T}, \quad \sqrt{\frac{T}{\hbar\left|\omega_{\pi}^{\prime \prime}\right|}} \ll \mp(q-\pi) \ll \pi .
$$

We now solve the Fokker-Planck equation (6) with the boundary conditions (8) to determine the steady-state phonon distribution $P(q)$ at $|q-\pi| \lesssim \sqrt{T / \hbar\left|\omega_{\pi}^{\prime \prime}\right|}$. The solution is rather straightforward and analogous to that for the distribution function of holes near the bottom of the conduction band of weakly interacting electrons [11]. It interpolates smoothly between the Boltzmann functions (8) and corresponds to a finite but exponentially 
small probability current

$$
J=u B\left(\frac{\pi \hbar^{3}\left|\omega_{\pi}^{\prime \prime}\right|}{2 T^{3}}\right)^{1 / 2} e^{-\hbar \omega_{\pi} / T} .
$$

The non-vanishing value of $J$ means that in unit time $(N / 2 \pi) J$ phonons increase their momentum and leave the first Brillouin zone through point $q=\pi$. (Here $N$ is the total number of electrons in the Wigner crystal.) Each such event is an Umklapp process resulting in the decrease of the quasimomentum $Q$ of the phonon system by $2 \pi$. We therefore conclude that at non-zero phonon velocity $u$ the phonon scattering events result in $\dot{Q}=-N J$. Comparing this result with the total quasimomentum of the phonons $Q=\pi N u T^{2} / 3 s^{3}$, easily computed using the distribution (5), we find the relaxation rate $\tau^{-1}=-\dot{Q} / Q$ in the form

$$
\tau^{-1}=3 B\left(\frac{\hbar s}{T}\right)^{3}\left(\frac{\hbar\left|\omega_{\pi}^{\prime \prime}\right|}{2 \pi T}\right)^{1 / 2} e^{-\hbar \omega_{\pi} / T}
$$

As expected, the relaxation rate shows activated temperature dependence, with activation temperature given by the Debye energy of the phonons $\hbar \omega_{\pi}$. However, to fully determine the temperature dependence of the prefactor, one has to calculate the diffusion coefficient $B$ of phonons in momentum space.
It is convenient to treat the scattering of phonons using the second quantization of the Hamiltonian (1) whereby the displacements and momenta of the particles are expressed in terms of the phonon destruction and creation operators $b_{q}$ and $b_{q}^{\dagger}$ as

$$
\begin{aligned}
& u_{l}=\sum_{q} \sqrt{\frac{\hbar}{2 m N \omega_{q}}}\left(b_{q}+b_{-q}^{\dagger}\right) e^{i q l}, \\
& p_{l}=-i \sum_{q} \sqrt{\frac{\hbar m \omega_{q}}{2 N}}\left(b_{q}-b_{-q}^{\dagger}\right) e^{i q l} .
\end{aligned}
$$

The quadratic Hamiltonian (2) then takes the standard form

$$
H_{0}=\sum_{q} \hbar \omega_{q}\left(b_{q}^{\dagger} b_{q}+1 / 2\right)
$$

The coupling of phonons is described by the anharmonic corrections to $H_{0}$, which are easily obtained by expanding the full Hamiltonian (1) to higher orders in the displacements $u_{l}$. To find the leading contribution to phonon scattering, we will need to account only for the cubic and quartic anharmonisms, $H \approx H_{0}+U^{(3)}+U^{(4)}$, with the respective perturbations taking forms

$$
\begin{aligned}
U^{(3)} & =\frac{-i}{3 \sqrt{N}}\left(\frac{\hbar}{2 m}\right)^{3 / 2} \sum_{q_{1}, q_{2}} \frac{f_{3}\left(q_{1}, q_{2}\right)}{\sqrt{\omega_{q_{1}} \omega_{q_{2}} \omega_{q_{1}+q_{2}}}}\left(b_{q_{1}}+b_{-q_{1}}^{\dagger}\right)\left(b_{q_{2}}+b_{-q_{2}}^{\dagger}\right)\left(b_{-q_{1}-q_{2}}+b_{q_{1}+q_{2}}^{\dagger}\right), \\
U^{(4)} & =\frac{\hbar^{2}}{48 m^{2} N} \sum_{q_{1}, q_{2}, q_{3}} \frac{f_{4}\left(q_{1}, q_{2}, q_{3}\right)}{\sqrt{\omega_{q_{1}} \omega_{q_{2}} \omega_{q_{3}} \omega_{q_{1}+q_{2}+q_{3}}}}\left(b_{q_{1}}+b_{-q_{1}}^{\dagger}\right)\left(b_{q_{2}}+b_{-q_{2}}^{\dagger}\right)\left(b_{q_{3}}+b_{-q_{3}}^{\dagger}\right)\left(b_{-q_{1}-q_{2}-q_{3}}+b_{q_{1}+q_{2}+q_{3}}^{\dagger}\right) .
\end{aligned}
$$

Here the functions $f_{3}$ and $f_{4}$ are defined as

$f_{3}\left(q_{1}, q_{2}\right)=\sum_{l=1}^{\infty} V_{l}^{(3)}\left\{\sin \left[\left(q_{1}+q_{2}\right) l\right]-\sin \left(q_{1} l\right)-\sin \left(q_{2} l\right)\right\}$

and

$$
\begin{aligned}
f_{4}\left(q_{1}, q_{2}, q_{3}\right)= & \sum_{l=1}^{\infty} V_{l}^{(4)}\left\{1-\cos \left(q_{1} l\right)-\cos \left(q_{2} l\right)\right. \\
& -\cos \left(q_{3} l\right)-\cos \left[\left(q_{1}+q_{2}+q_{3}\right) l\right] \\
& +\cos \left[\left(q_{1}+q_{2}\right) l\right]+\cos \left[\left(q_{1}+q_{3}\right) l\right] \\
& \left.+\cos \left[\left(q_{2}+q_{3}\right) l\right]\right\} .
\end{aligned}
$$

The rate of two-phonon scattering processes shown in Fig. 1] in which a phonon $q_{1}$ moves to the state $q_{1}^{\prime}$, is given by the golden rule expression

$$
\begin{aligned}
W_{q_{1}, q_{1}^{\prime}}= & \frac{2 \pi}{\hbar^{2}} \sum_{q_{2}, q_{2}^{\prime}}\left|t_{q_{1}, q_{2} \rightarrow q_{1}^{\prime}, q_{2}^{\prime}}\right|^{2} N_{q_{2}}\left(N_{q_{2}^{\prime}}+1\right) \\
& \times \delta_{q_{1}+q_{2}, q_{1}^{\prime}+q_{2}^{\prime}} \delta\left(\omega_{q_{1}}+\omega_{q_{2}}-\omega_{q_{1}^{\prime}}-\omega_{q_{2}^{\prime}}\right) .
\end{aligned}
$$

The scattering of two phonons $q_{1}$ and $q_{2}$ into $q_{1}^{\prime}$ and $q_{2}^{\prime}$, can be accomplished in the first order in the quartic anharmonism $U^{(4)}$. Alternatively, the same scattering process can be realized in second order in the cubic anharmonism $U^{(3)}$. Simple power counting shows that in both cases the resulting amplitude is proportional to $\hbar^{2}$, i.e., one has to account for both contributions. The actual calculation is straightforward and results in the scattering matrix element in the form

$$
t_{q_{1}, q_{2} \rightarrow q_{1}^{\prime}, q_{2}^{\prime}}=\frac{\hbar^{2}}{m^{3} N} \frac{\Lambda}{\left(\omega_{q_{1}} \omega_{q_{2}} \omega_{q_{1}^{\prime}} \omega_{q_{2}^{\prime}}\right)^{1 / 2}},
$$


where

$$
\begin{aligned}
\Lambda= & -\frac{f_{3}\left(q_{1}, q_{2}\right) f_{3}\left(q_{1}^{\prime}, q_{2}^{\prime}\right)}{\omega_{q_{1}+q_{2}}^{2}-\left(\omega_{q_{1}}+\omega_{q_{2}}\right)^{2}}+\frac{f_{3}\left(q_{2},-q_{1}^{\prime}\right) f_{3}\left(q_{1},-q_{2}^{\prime}\right)}{\omega_{q_{2}-q_{1}^{\prime}}^{2}-\left(\omega_{q_{2}}-\omega_{q_{1}^{\prime}}\right)^{2}} \\
& +\frac{f_{3}\left(q_{1},-q_{1}^{\prime}\right) f_{3}\left(q_{2},-q_{2}^{\prime}\right)}{\omega_{q_{2}-q_{2}^{\prime}}^{2}-\left(\omega_{q_{2}}-\omega_{q_{2}^{\prime}}\right)^{2}}+\frac{m}{2} f_{4}\left(q_{1}, q_{2},-q_{1}^{\prime}\right) . \quad(20)
\end{aligned}
$$

To find the diffusion constant $B\left(q_{1}\right)$ of phonons in momentum space, one notes that the momentum $q_{2}$ is limited by the occupation number $N_{q_{2}}$ in Eq. (18), resulting in $\left|q_{2}\right| \sim\left|q_{2}^{\prime}\right| \sim T / \hbar s \ll \pi$. Thus one can expand $\Lambda$ in powers of $\delta q=q_{1}^{\prime}-q_{1}=q_{2}-q_{2}^{\prime}$. The expansion starts with a quadratic term, $\Lambda \propto(\delta q)^{2}$. The proportionality constant depends on the specific model of the interaction potential $V(x)$. In the most interesting case of Coulomb potential $V(x)=e^{2} /|x|$ our treatment is complicated by the fact that the phonon speed $s$ diverges logarithmically. In practice, however, the Coulomb potential is always screened at large distances by remote gates. In this case, $\Lambda$ can be found analytically. For simplicity, we also limit ourselves to the most important case of $q_{1}=\pi$ and find

$$
\Lambda=\frac{21 \zeta(3)}{32} \frac{m e^{2}}{a^{5}}(\delta q)^{2},
$$

where $\zeta(x)$ is the Riemann's zeta function. Combining this result with Eqs. (19), (18), and (7), we find

$$
B=\chi T^{5}, \quad \chi=\frac{21 \pi^{3} \zeta(3)}{20} \frac{e^{2}}{\hbar^{3} m^{3} a^{7} s^{8}} .
$$

One can now substitute this result into Eq. (10) to obtain the full expression for the relaxation rate of the phonon system in a Wigner crystal. In the specific case of Coulomb interaction, the Debye frequency $\omega_{\pi}=\left(7 \zeta(3) e^{2} / m a^{3}\right)^{1 / 2}$, parameter $\omega_{\pi}^{\prime \prime}=$ $-2 \ln 2\left(e^{2} / 7 \zeta(3) m a^{3}\right)^{1 / 2}$, and the speed of phonons is $s=\left(2 e^{2} \ln (d / a) / m a^{3}\right)^{1 / 2}$, where $d$ is the distance to the gate.

Although the expression (22) is derived for Coulomb interaction, the temperature dependence $B \propto T^{5}$ is valid for any long-range repulsive potential. An interesting exception is the case of strong inverse-square repulsion, $V(x)=\gamma / x^{2}$, with $\gamma \gg \hbar^{2} / m$. This form of repulsive potential corresponds to the Calogero-Sutherland model, which is exactly solvable due to the presence of an infinite number of integrals of motion. As a result, one expects that scattering of excitations preserves their momenta, and no diffusion in momentum space should be possible. Indeed, we have been able to show that the coefficient $\chi$ in Eq. (22) vanishes for inverse-square repulsion. We have also verified that the expression (20) for $\Lambda$, and thus the phonon scattering amplitude (19), vanish in this case. Scattering of a massive particle off an acoustic phonon, Fig. 1, was recently discussed in the context of quantum decay of dark solitons in one dimensional Bose systems
[14]. In analogy with our observation, their decay rate vanishes in the integrable (Lieb-Liniger) case.

The expression (10) for the relaxation rate of phonon system in a one-dimensional Wigner crystal with the diffusion constant (22) is the main result of this paper. To illustrate its significance we now briefly discuss the effect of the phonon relaxation upon the conductance of a strongly-interacting quantum wire. A more rigorous treatment will be presented elsewhere.

The equilibration of phonons in a one-dimensional Wigner crystal is analogous to electron equilibration in the limit of weak interactions. In the latter case, the excitations are holes/particles created by transferring electrons to/from a Fermi point. The momentum of each excitation is thus measured from the nearest Fermi point. Equilibration processes include backscattering of holes near the bottom of the band. Since the momenta of rightand left-moving holes are measured from different Fermi points, each backscattering event changes the momentum of the excitations by $2 p_{F}=2 \pi / a$, in analogy with the quasimomentum change $\Delta Q=2 \pi$ when a phonon in a Wigner crystal crosses the edge of the Brillouin zone. We thus conclude that $\dot{Q}$ can be interpreted as the rate of backscattering of right-moving electrons, $\dot{N}^{R}=-\dot{Q} / 2 \pi$.

This relation provides for a way to observe the phonon equilibration by measuring conductance of a strongly interacting wire connected to non-interacting leads. Negative value of $\dot{N}^{R}$ means that some of the right-moving electrons entering the wire from the left return to the same lead, thereby reducing the conductance [6, 9, 11]. Adapting the calculation of Ref. [11] to the case strong interactions, one finds

$$
G=\frac{e^{2}}{h}\left[1-L \frac{B}{a}\left(\frac{\hbar^{3}\left|\omega_{\pi}^{\prime \prime}\right|}{8 \pi T^{3}}\right)^{1 / 2} e^{-\hbar \omega_{\pi} / T}\right]
$$

Although the correction to the conductance is exponentially small, it grows with the length of the wire $L$. The result (23) applies to relatively short wires, $L \ll l_{\text {eq }} \propto$ $e^{\hbar \omega_{\pi} / T}$, c.f. Ref. [1].

To summarise, we have studied the relaxation of the distribution function of phonons in a one-dimensional spinless Wigner crystal. Full equilibration of phonons requires Umklapp processes, resulting in an exponentially small relaxation rate. The preexponential factor in the resulting expression (10) scales as $T^{3 / 2}$. In the integrable case of inverse-square interactions the relaxation rate vanishes.

The authors are grateful to D. M. Gangardt and A. Kamenev for helpful discussions. This work was supported by the U.S. Department of Energy under Contract Nos. DE-AC02-06CH11357, DE-FG02-07ER46452, and DE-FG02-06ER46311. 
[1] F. D. M. Haldane, J. Phys. C 14, 2585 (1981).

[2] T. Giamarchi, Quantum Physics in One Dimension, (Clarendon Press, Oxford, 2004).

[3] C. L. Kane and M. P. A. Fisher, Phys. Rev. B 46, 15233 (1992).

[4] A. Furusaki and N. Nagaosa, Phys. Rev. B 47, 4631 (1993).

[5] M. Pustilnik, M. Khodas, A. Kamenev, and L. I. Glazman, Phys. Rev. Lett. 96, 196405 (2006).

[6] A. M. Lunde, K. Flensberg, and L. I. Glazman, Phys. Rev. B 75, 245418 (2007).

[7] G. A. Fiete, Rev. Mod. Phys. 79, 801 (2007).
[8] J. S. Meyer and K. A. Matveev, J. Phys: Condens. Matter 21, 023203 (2009).

[9] J. Rech, T. Micklitz, and K. A. Matveev, Phys. Rev. Lett. 102, 116402 (2009)

[10] M. Pustilnik, E. G. Mishchenko, L. I. Glazman, and A.V. Andreev, Phys. Rev. Lett. 91, 126805 (2003).

[11] T. Micklitz, J. Rech, K. A. Matveev, Phys. Rev. B 81, 115313 (2010).

[12] For a more detailed discussion of the relation between one-dimensional Wigner crystal and Luttinger liquid see review [8] and references therein.

[13] E. M. Lifshitz and L. P. Pitaevskii, Physical kinetics, (Pergamon Press, Oxford, 1981), §67.

[14] D. M. Gangardt and A. Kamenev, arXiv:0908.4513 